\title{
A Simple Approach to Great Circle Sailing: The COFI Method
}

\author{
Chih-Li Chen, Pin-Fang Liu and Wei-Ting Gong \\ (Merchant Marine Department, National Taiwan Ocean University) \\ (E-mail: clchen@mail.ntou.edu.tw)
}

\begin{abstract}
An approach formulated by vector algebra is proposed to deal with great circle sailing problems. Using the technique of the fixed coordinates system and relative longitude concept, derivations of formulae for this approach are simpler than those of the conventional methods. Due to fixing the initial great circle course, the great circle track (GCT) is determined. Since the course is fixed (known as "COFI" in this paper), the proposed approach, which we have named the "COFI method", can directly calculate the waypoints along the GCT. It is considered that the COFI method is a more understandable and straightforward method to solve waypoint problems than older approaches in the literature. Based on the COFI method, a program has been developed for the navigator. In addition, the spherical triangle method with respect to the equator crossing point (STM-E) is developed by supplemental theorem. Several examples are demonstrated to validate the proposed COFI method and STM-E.
\end{abstract}

\section{KEY WORDS}

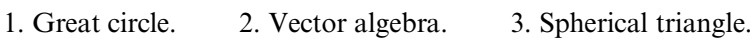

Submitted: 2 July 2013. Accepted: 29 October 2013. First published online: 27 November 2013.

1. INTRODUCTION. The shortest distance between any two non-antipodal points on a sphere's surface is the only great circle track (GCT) passing through them. The Earth can be considered as a sphere for obtaining the waypoints on the GCT by using great circle sailing (GCS). However, because of the Earth's rotation, the Earth is approximately an oblate spheroid (or ellipsoid of revolution). Consequently, the Mercator chart or an Electronic Chart Display and Information System (ECDIS) usually uses the WGS 84 (World Geodetic System ellipsoid of 1984). A great circle other than a meridian or the equator is a curved line whose true direction changes continually, thus navigators do not usually attempt to follow it exactly. Instead, they select a number of waypoints along the GCT, construct rhumb lines between the waypoints on the Mercator chart or in the ECDIS, and then steer along these rhumb lines (Bowditch, 2002). In practical navigation, the waypoints on the GCT are entered into the ECDIS, GPS, or a fully integrated navigation system. Then, the vessel is programmed to follow the GCT by sailing from waypoint to waypoint by rhumb line, with allowances made for wind and current effects. 
When the Earth is regarded as a sphere, the navigator has to give initial conditions for obtaining the waypoints along the GCT. Generally speaking, these given initial conditions include: giving the longitudes of the waypoints to obtain their latitudes (Condition 1); giving the great circle distances to yield the latitudes and longitudes of the waypoints (Condition 2). Once all the waypoints on the GCT are available, the navigator needs to take the Earth as an oblate spheroid for practical navigation. Because the GCT is composed of legs of rhumb lines, the course and distance of the rhumb line between two adjacent waypoints can be determined by using the Mercator sailing.

The spherical triangle method (STM) with a reference point at the vertex (called STM-V) has been developed to solve the waypoints on the GCT for many years. It can handle the waypoints problem under Conditions 1 or 2 (Holm, 1972; Bowditch, 1981, 2002; Keys, 1983; Cutler, 2004; Chen et al., 2004). The advantage of this method is that the solved formulae are simplified because the method uses Napier's rules of right-angled spherical triangles. Finding the equator crossing point of the GCT is easier than finding the vertex of the GCT. In addition, when the supplemental theorem is introduced, the right-angled spherical triangles can be converted into quadrantal spherical triangles (Clough-Smith, 1966). Therefore, a method with the reference point at the equator crossing point (called STM-E) should be available. The STM-E has the same advantage as the STM-V and can also deal with the waypoints problem under Condition 1 or 2. However, a common disadvantage of the STM-V and STM-E is that the reference point should be determined in advance. Owing to this disadvantage, both methods are usually considered as a type of indirect approach (IA).

To overcome this shortcoming, some researchers take the departure point as the reference point. This means one can replace the Greenwich meridian by the meridian of the departure point and this is usually called the relative longitude concept. Similarly, Jofeh (1981) constructed a linear equation (LE) of the GCT, which appears as a straight line on the polar gnomonic chart. According to his method, the latitudes of the waypoints are determined only under Condition 1. Unfortunately, when the departure and destination points are located in different hemispheres, the method fails. Later, Miller et al. (1991) first used the technique of the fixed coordinates system to construct a vector expression of the waypoints and then adopted linear combination (LC) of a vector basis to formulate another vector expression. Comparing the components of the different vector expressions yields three key formulae, that is, five-parts formula (x-component), five-parts formula (y-component) and side cosine law (z-component). Then, a combination of the five-parts formula (x-component) and the side cosine law (z-component) can handle the waypoints problem but only under Condition 2. Thereafter, Chen et al. (2004) first combined the technique of the fixed coordinates system with the relative longitude concept (FCRL) and then proposed the great circle equation method (GCEM), in which the great circle equation is formulated by vector algebra. It is found that the GCEM can deal with the waypoints problem only under Condition 1. In addition, like Miller et al. (1991), Nastro and Tancredi (2010) adopted linear combination (LC) of a different vector basis with the FCRL, also reaching three key formulae. That is, five-parts formula (x-component), sine law (y-component) and side cosine law (z-component). Then, the sine law divided by the five-parts formula obtains the four-parts formula. A combination of this formula and the yielded side cosine law can handle the waypoints problem but only under Condition 2. However, tedious derivations make their solutions hard to understand. 
Table 1. A comparison of different methods for solving the GCS.

\begin{tabular}{llll}
\hline Authors & \multicolumn{1}{c}{ Category } & Condition 1 & Condition 2 \\
\hline Holm, 1972 & IA (STM-V) & available & NA \\
Jofeh, 1981 & DA (LE) & Available* & NA \\
Bowditch, 1981 and 2002 & IA (STM-V) & available & available \\
Keys, 1983 & IA (STM-V) & available & available \\
Miller et al., 1991 & DA (LC) & NA & available \\
Cutler, 2004 & IA (STM-V) & available & available \\
Chen et al., 2004 & IA (STM-V) & available & available \\
Nastro and Tancredi, 2010 & DA (GCEM) & available & NA \\
Chen et al., 2013 (the current paper) & DA (LC) & NA & available \\
& IA (STM-E) & available & available \\
& DA (COFI) & NA & available \\
\hline
\end{tabular}

* This method fails when departure and destination points are located in different hemispheres.

In contrast to the IA, the methods mentioned above all belong to a type of direct approach (DA). The comparison of the mentioned methods is listed in Table 1. In this table, it is found that the DA can solve the waypoints problem under either Condition 1 or Condition 2; while the IA can deal with the problems under both Conditions 1 and 2.

To overcome the complex derivations (Miller et al., 1991; Nastro and Tancredi, 2010), the concise derivation of the formulae by using multiple products of vector algebra (VA-MP) with the FCRL is proposed to solve the waypoints problem under Condition 2. Once the initial great circle course is fixed (COFI), the GCT can be determined. With this characteristic, the proposed approach is named the "COFI method". Further, to tackle the waypoints problem covering Conditions 1 and 2, a program, based on the COFI method and the simplified GCEM, has been developed for the practical navigator. In addition, because the STM-E can deal with the waypoints problem under two given conditions, derivations of the method are also included in this article.

Theoretical backgrounds of the STM-E, the COFI method and the simplified GCEM are presented in Section 2. Section 3 describes the computation procedures of the COFI method and the simplified GCEM. Validated examples are given in Section 4. Finally, the work is summarised and concluded in Section 5.

\section{THEORETICAL BACKGROUNDS.}

2.1. Deriving Formulae for the STM-E. As mentioned in the previous section, the supplemental theorem can be used to derive the formulae of the STM-E for solving the waypoints problem. The supplemental theorem describes (Clough-Smith, 1966):

"The angles in the polar triangle are supplements of the corresponding sides in the primitive triangle, and the sides in the polar triangle are supplements of the corresponding angles in the primitive triangle."

Due to this property, those formulae used in right-angled spherical triangles can also work in quadrantal spherical triangles. In addition, because the great circle arc from the equator crossing point to the pole should be $90^{\circ}$, the equator crossing point, 


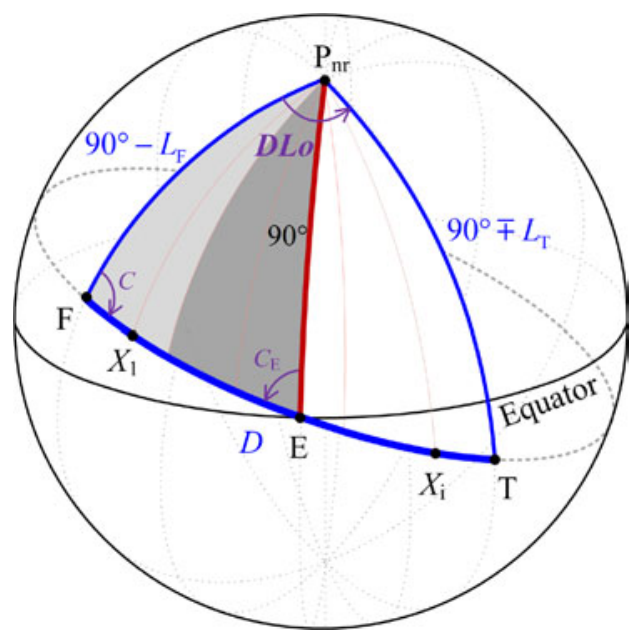

Figure 1. An illustration of the STM-E for solving the problem of GCS.
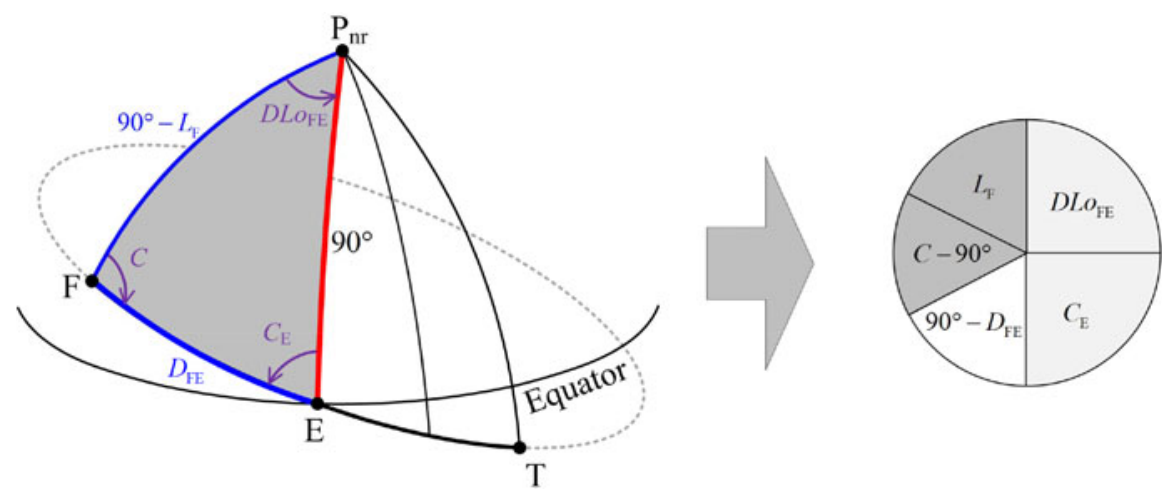

Figure 2. An illustration of finding the equator crossing point on the GCT by using Napier's rule of quadrantal spherical triangles.

the pole nearer the departure and waypoints along the GCT can form numerous quadrantal spherical triangles as shown in Figure 1. Consequently, the solving steps of the STM-E are presented as follows. All the symbols used below are listed in the Appendix.

Step 1. Finding the great circle distance $(D)$ and the initial great circle course angle $(C)$ as shown in Figure 1. The great circle distance and the initial course angle can be calculated by the side cosine law and the four-parts formula of the spherical trigonometry, respectively as (Chen et al., 2004):

$$
\begin{gathered}
\cos D=\sin L_{\mathrm{F}} \sin L_{\mathrm{T}}+\cos L_{\mathrm{F}} \cos L_{\mathrm{T}} \cos D L o, \\
\tan C=\frac{\sin D L o}{\left(\cos L_{\mathrm{F}} \tan L_{\mathrm{T}}\right)-\left(\sin L_{\mathrm{F}} \cos D L o\right)} .
\end{gathered}
$$

Step 2. Finding the longitude of the equator crossing point, $\lambda_{\mathrm{E}}$, as shown in Figure 2. By using Napier's rules of quadrantal spherical triangles, the following two formulae 


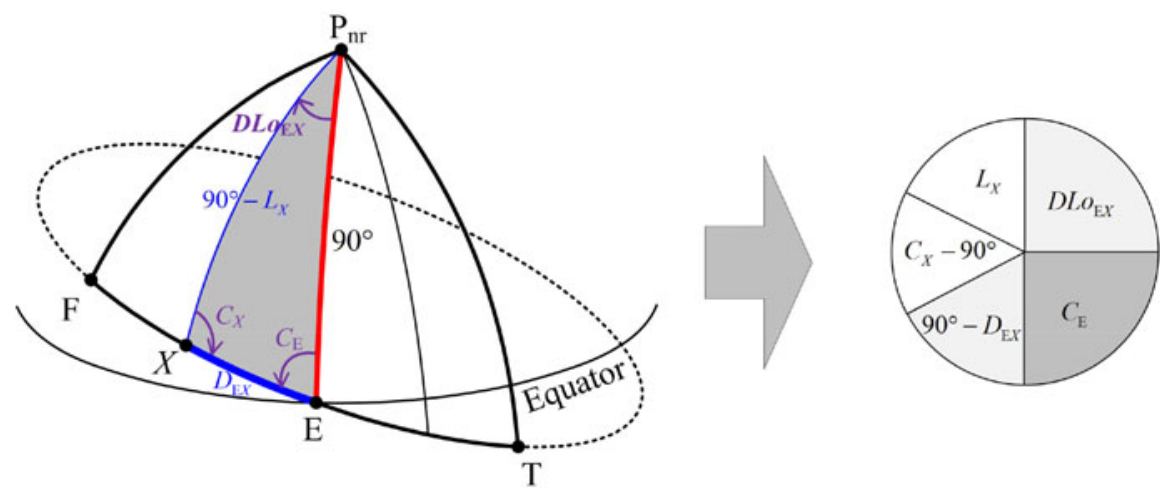

Figure 3. An illustration of finding the waypoints on the GCT by using Napier's rule of quadrantal spherical triangles.

can be yielded as:

$$
\begin{gathered}
\tan D L o_{\mathrm{FE}}=-\sin L_{\mathrm{F}} \tan C, \\
\sin C_{\mathrm{E}}=\cos L_{\mathrm{F}} \sin C .
\end{gathered}
$$

Note that if $D L o_{\mathrm{FE}}$ has the same name as $D L o$ (i.e. both West or both East) and $\lambda_{\mathrm{F}}$ is available, $\lambda_{\mathrm{E}}$ can be obtained by Equation (3). In addition, $C_{\mathrm{E}}$, can be obtained by Equation (4) and it will be used in the following step.

Step 3. Finding the latitudes and longitudes of the waypoints along the GCT is shown in Figure 3. Since only $C_{\mathrm{E}}$ is available, the given condition is necessary for obtaining the waypoints. By using Napier's rules of quadrantal spherical triangles, we can yield the following formulae under both Conditions 1 and 2.

Condition 1. When $\lambda_{X}$ is given, $D L o_{\mathrm{E} X}$ can be obtained. Then, $L_{X}$ can be calculated from the following formula

$$
\tan L_{X}= \pm \cot C_{\mathrm{E}} \sin D L o_{\mathrm{E} X}
$$

Note that if $D L o_{\mathrm{E} X}$ is the contrary name to $D L o$ (i.e. one East and one West), the right-hand side of Equation (5) should take the positive sign. It means $L_{X}$ and $L_{F}$ are located in the same hemisphere. Conversely, if $D L o_{\mathrm{E} X}$ has the same name as $D L o$, the right-hand side of Equation (5) should be treated as negative sign. It means $L_{X}$ and $L_{F}$ are located in different hemispheres.

Condition 2. When the $D_{\mathrm{E} X}$ is given, the waypoints can be obtained from the formulae,

$$
\begin{gathered}
\sin L_{X}=\cos C_{\mathrm{E}} \sin D_{\mathrm{E} X}, \\
\tan D L o_{\mathrm{E} X}=\sin C_{\mathrm{E}} \tan D_{\mathrm{E} X} .
\end{gathered}
$$

In Equation (6), note that when $L_{X}$ is smaller than $L_{F}$, the waypoints are on the GCT. This means $L_{X}$ and $L_{F}$ are located in the same hemisphere. Thus, $L_{X}$ is taken as the positive. Similarly, when $L_{X}$ is smaller than $L_{T}$, the waypoints are on the GCT but $L_{X}$ and $L_{T}$ are located in different hemispheres. In this regard, $L_{X}$ is taken as the negative. In Equation (7), if the value of $\tan D L o_{\mathrm{E} X}$ is negative, $\left(180^{\circ}-D L o_{\mathrm{E} X}\right)$ should replace $\left(-D L o_{\mathrm{E} X}\right.$ ) for satisfying the definition of $D L o_{\mathrm{E} X}$. As for the designated 


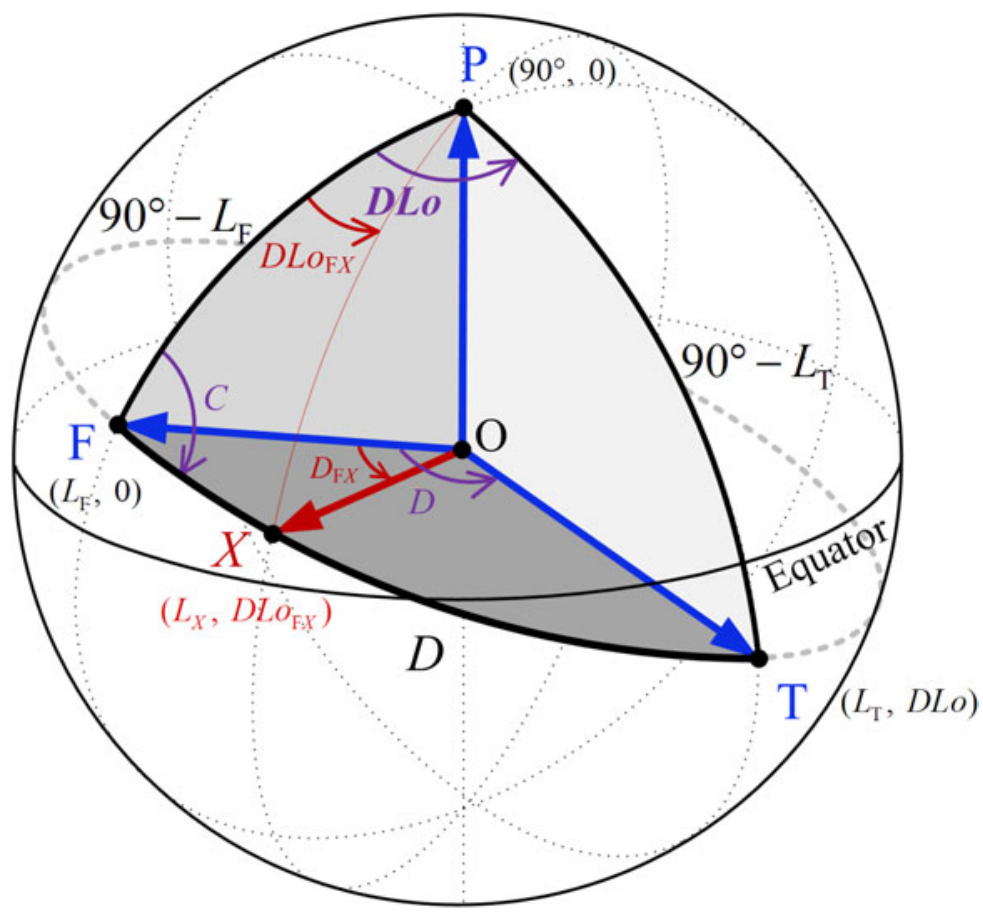

Figure 4. An illustration of four position vectors.

east or west of $D L o_{\mathrm{E} X}$, it depends on the sign of $L_{X}$. If $L_{X}$ is positive, $D L o_{\mathrm{E} X}$ is contrary name to $D L o$. However, if $L_{X}$ is negative, $D L o_{\mathrm{E} X}$ has the same name as $D L o$. Obviously, too many judgments of sign conventions arise in the solving procedures of the STM-E and this makes use of this method hard work for the navigator. However, the STM-E method still offers a way to solve the waypoints problem.

2.2. Deriving Formulae for the COFI method. As the Earth is treated as a unitary sphere, the vector expression of any point $G(L, \lambda)$ on the Earth's surface in a Cartesian coordinates system can be written as:

$$
\overrightarrow{\mathbf{G}}=[\cos L \cos \lambda, \cos L \sin \lambda, \sin L], L=\left[-\frac{\pi}{2}, \frac{\pi}{2}\right], \lambda=[0,2 \pi) .
$$

To avoid an additional judgment of sign convention, the technique of the fixed coordinates system is first considered, that is, the north latitude is treated as a positive value and the south latitude is taken as a negative one. As shown in Figure 4, introducing the relative longitude concept, the unit vectors of the North Pole $(\mathrm{P})$, the departure $(\mathrm{F})$, the destination $(\mathrm{T})$ and the waypoints $(X)$ on a GCT can be expressed as:

$$
\begin{gathered}
\overrightarrow{\mathbf{P}}=[0,0,1], \\
\overrightarrow{\mathbf{F}}=\left[\cos L_{\mathrm{F}}, 0, \sin L_{\mathrm{F}}\right], \\
\overrightarrow{\mathbf{T}}=\left[\cos L_{\mathrm{T}} \cos D L o, \cos L_{\mathrm{T}} \sin D L o, \sin L_{\mathrm{T}}\right], \\
\overrightarrow{\mathbf{X}}=\left[\cos L_{X} \cos D L o_{\mathrm{F} X}, \cos L_{X} \sin D L o_{\mathrm{F} X}, \sin L_{X}\right] .
\end{gathered}
$$


When the vector algebra is introduced, derivations of the formulae used for the COFI will be simpler and clearer than those of the DA. Therefore, we adopt multiple products of the vector algebra to yield the great circle distance, the initial course and the waypoints on the GCT (Spiegel, 2009; Chen et al., 2004).

2.2.1. Obtaining the great circle distance. As shown in Figure 4, there are two ways to obtain the great circle distance. One is to yield the great circle arc $(D)$ of the spherical triangle by the dot product of two unit vectors. That is,

$$
\begin{aligned}
\overrightarrow{\mathbf{F}} \cdot \overrightarrow{\mathbf{T}} & =\cos D \\
& =\cos L_{\mathrm{F}} \cos L_{\mathrm{T}} \cos D L o+\sin L_{\mathrm{F}} \sin L_{\mathrm{T}},
\end{aligned}
$$

in which the first row of the above equation uses the geometric definition of vector product, while the second row uses the algebraic operation of vector product.

Another method is to adopt the dot product of two normal vectors, $(\overrightarrow{\mathbf{P}} \times \overrightarrow{\mathbf{T}})$ and $(\overrightarrow{\mathbf{P}} \times \overrightarrow{\mathbf{F}})$, to yield the dihedral angle $(D L o)$ of the spherical triangle. Therefore,

$$
\begin{aligned}
(\overrightarrow{\mathbf{P}} \times \overrightarrow{\mathbf{T}}) \cdot(\overrightarrow{\mathbf{P}} \times \overrightarrow{\mathbf{F}}) & =\cos L_{\mathrm{T}} \cos L_{\mathrm{F}} \cos D L o \\
& =\left|\begin{array}{ll}
(\overrightarrow{\mathbf{P}} \cdot \overrightarrow{\mathbf{P}}) & (\overrightarrow{\mathbf{P}} \cdot \overrightarrow{\mathbf{F}}) \\
(\overrightarrow{\mathbf{T}} \cdot \overrightarrow{\mathbf{P}}) & (\overrightarrow{\mathbf{T}} \cdot \overrightarrow{\mathbf{F}})
\end{array}\right|=\cos D-\sin L_{\mathrm{F}} \sin L_{\mathrm{T}}
\end{aligned}
$$

Similarly, two rows of Equation (14) represent the same mathematical meanings as those of Equation (13). After arranging Equations (13) or (14), we can write the same governing equation as:

$$
\cos D=\sin L_{\mathrm{F}} \sin L_{\mathrm{T}}+\cos L_{\mathrm{F}} \cos L_{\mathrm{T}} \cos D L o
$$

Equation (15) is the well-known side cosine law of spherical trigonometry.

2.2.2. Obtaining the initial great circle course angle. To yield the initial great circle course angle, that is, the dihedral angle $(C)$ of the spherical triangle, we adopt the dot product of two normal vectors, $(\overrightarrow{\mathbf{F}} \times \overrightarrow{\mathbf{P}})$ and $(\overrightarrow{\mathbf{F}} \times \overrightarrow{\mathbf{T}})$. Hence,

$$
\begin{aligned}
(\overrightarrow{\mathbf{F}} \times \overrightarrow{\mathbf{P}}) \cdot(\overrightarrow{\mathbf{F}} \times \overrightarrow{\mathbf{T}}) & =\cos L_{\mathrm{F}} \sin D \cos C \\
& =\sin L_{\mathrm{T}}-\sin L_{\mathrm{F}} \cos D,
\end{aligned}
$$

in which the first row uses the geometric definition of vector products, while the second row uses the algebraic operation of vector products. Rearranging Equation (16) obtains the governing equation as:

$$
\cos C=\frac{\sin L_{\mathrm{T}}-\sin L_{\mathrm{F}} \cos D}{\cos L_{\mathrm{F}} \sin D} .
$$

Equation (17) is another form of the side cosine law of the spherical trigonometry.

2.2.3. Obtaining the latitudes of waypoints along the GCT. As the initial great circle course is fixed, the GCT can be determined. Then, giving the great circle distance from the departure point (Condition 2) we can obtain every waypoint along the GCT. Replacing the parameter vector $\overrightarrow{\mathbf{T}}$ of Equation (16) by the variable 
vector $\overrightarrow{\mathbf{X}}$ yields

$$
\begin{aligned}
(\overrightarrow{\mathbf{F}} \times \overrightarrow{\mathbf{P}}) \cdot(\overrightarrow{\mathbf{F}} \times \overrightarrow{\mathbf{X}}) & =\cos L_{\mathrm{F}} \sin D_{\mathrm{F} X} \cos C \\
& =\sin L_{X}-\sin L_{\mathrm{F}} \cos D_{\mathrm{F} X} .
\end{aligned}
$$

Rearranging Equation (18) obtains

$$
\sin L_{X}=\sin L_{\mathrm{F}} \cos D_{\mathrm{F} X}+\cos L_{\mathrm{F}} \sin D_{\mathrm{F} X} \cos C .
$$

The above equation is also the side cosine law of the spherical trigonometry.

2.2.4. Obtaining the longitudes of waypoints along the GCT. Replacing parameter vector $\overrightarrow{\mathbf{T}}$ of Equation (14) by variable $\overrightarrow{\mathbf{X}}$ vector yields

$$
\begin{aligned}
(\overrightarrow{\mathbf{P}} \times \overrightarrow{\mathbf{X}}) \cdot(\overrightarrow{\mathbf{P}} \times \overrightarrow{\mathbf{F}}) & =\cos L_{X} \cos L_{\mathrm{F}} \cos D L o_{\mathrm{F} X} \\
& =\cos D_{\mathrm{F} X}-\sin L_{\mathrm{F}} \sin L_{X} .
\end{aligned}
$$

Rearranging Equation (20), we have

$$
\cos D L o_{\mathrm{F} X}=\frac{\cos D_{\mathrm{F} X}-\sin L_{\mathrm{F}} \sin L_{X}}{\cos L_{\mathrm{F}} \cos L_{X}} .
$$

The above equation is another form of the side cosine law of spherical trigonometry.

Note that Equations (19) and (21) are a set of the governing equations to obtain the latitudes and longitudes of the waypoints along the GCT under Condition 2. In summary, formulae used in the COFI method are only a form of the side cosine law of spherical trigonometry. Therefore, introducing the vector algebra into derivations of the COFI method makes this method simpler and clearer than the conventional approaches.

2.3. Reformulating the formulae used for the GCEM. To solve the waypoints problem covering Conditions 1 and 2, the GCEM and the COFI method should be combined for the practical navigator. Therefore, revisiting and simplifying the formulae of the GCEM are described as follows.

2.3.1. Revisiting the GCEM. Those formulae used for the GCEM are briefly revisited here (Chen et al., 2004). As shown in Figure 4, if three vectors are coplanar, the scalar triple product is equal to zero. That is,

$$
(\overrightarrow{\mathbf{F}} \times \overrightarrow{\mathbf{T}}) \cdot \overrightarrow{\mathbf{X}}=0
$$

Now, assuming

$$
\overrightarrow{\mathbf{F}} \times \overrightarrow{\mathbf{T}}=[a, b, c],
$$

and substituting Equations (10) and (11) into Equation (23) yield

$$
\begin{gathered}
a=-\sin L_{\mathrm{F}} \cos L_{\mathrm{T}} \sin D L o, \\
b=\sin L_{\mathrm{F}} \cos L_{\mathrm{T}} \cos D L o-\cos L_{\mathrm{F}} \sin L_{\mathrm{T}}, \\
c=\cos L_{\mathrm{F}} \cos L_{\mathrm{T}} \sin D L o .
\end{gathered}
$$

Finally, the Great Circle Equation can be formulated as

$$
a \cos L_{X} \cos D L o_{\mathrm{F} X}+b \cos L_{X} \sin D L o_{\mathrm{F} X}+c \sin L_{X}=0 .
$$


Note that Equation (27) implies the information of the great circle, for example, the waypoints along the GCT, the equator crossing point and the vertex.

1. The waypoints along the GCT: When $\lambda_{X}$ is given, $D L o_{\mathrm{F} X}$ can be obtained. Rearranging Equation (27) yields

$$
\tan L_{X}=\frac{a \cos D L o_{\mathrm{F} X}+b \sin D L o_{\mathrm{F} X}}{-c} .
$$

2. The equator crossing point: Since $L_{E}=0$, substituting it into Equation (28) yields

$$
\tan D L o_{\mathrm{FE}}=-\frac{a}{b}
$$

3. The vertex: When the vertex is the highest latitude for the great circle, the first derivative of Equation (28) must be zero. Therefore, we have

$$
\tan D L o_{\mathrm{FV}}=\frac{b}{a} \text {. }
$$

Substituting the above result into Equation (28) yields

$$
\tan L_{\mathrm{V}}=\frac{a \cos D L o_{\mathrm{FV}}+b \sin D L o_{\mathrm{FV}}}{-c} .
$$

2.3.2. Simplifying formulae used for the GCEM. Because Equation (25) used for obtaining the parameter, $b$, is complex, we need to simplify it for a practical use. First, Equations (15) and (17) can be rewritten as

$$
\begin{gathered}
\cos D L o=\frac{\cos D-\sin L_{\mathrm{F}} \sin L_{\mathrm{T}}}{\cos L_{\mathrm{F}} \cos L_{\mathrm{T}}}, \\
\sin L_{\mathrm{T}}=\sin L_{\mathrm{F}} \cos D+\cos L_{\mathrm{F}} \sin D \cos C .
\end{gathered}
$$

Then, substituting Equations (32) and (33) into Equation (25) and rearranging it yield

$$
b=-\sin D \cos C \text {. }
$$

Introducing the sine law of spherical trigonometry, that is,

$$
\cos L_{\mathrm{T}} \sin D L o=\sin D \sin C,
$$

and substituting Equation (35) into Equations (24) and (26), respectively yield

$$
\begin{gathered}
a=-\sin L_{\mathrm{F}} \sin D \sin C, \\
c=\cos L_{\mathrm{F}} \sin D \sin C .
\end{gathered}
$$

Therefore, the concise formulae used for the simplified GCEM are as follows.

1. The waypoints along the GCT: Substituting Equations (34), (36) and (37) into Equation (28) and rearranging it yield

$$
\tan L_{X}=\frac{\cos C \sin D L o_{\mathrm{F} X}+\sin L_{\mathrm{F}} \sin C \cos D L o_{\mathrm{F} X}}{\cos L_{\mathrm{F}} \sin C} .
$$

2. The equator crossing point: Substituting Equations (34) and (36) into Equation (29) yields

$$
\tan D L o_{\mathrm{FE}}=-\sin L_{\mathrm{F}} \tan C
$$


3. The vertex: Substituting Equations (34) and (36) into Equation (30) yields

$$
\tan D L o_{\mathrm{FV}}=\frac{1}{\sin L_{\mathrm{F}} \tan C} .
$$

Further, substituting Equations (34), (36) and (37) into Equation (31) yields

$$
\tan L_{V}=\frac{\cos C \sin D L o_{\mathrm{F} V}+\sin L_{\mathrm{F}} \sin C \cos D L o_{\mathrm{F} V}}{\cos L_{\mathrm{F}} \sin C} .
$$

3. COMPUTATION PROCEDURES AND NUMERICAL PROGRAM. The great circle sailing problem is first to obtain the waypoints along the GCT. Then, the course and distance of the rhumb line between two adjacent waypoints can be determined by using the Mercator sailing. All required formulae used for the numerical program are listed as

$$
M=a_{e} \ln \left[\tan \left(45^{\circ}+\frac{L}{2}\right) \times\left(\frac{1-e \sin L}{1+e \sin L}\right)^{\frac{e}{2}}\right],
$$

in which $e=0 \cdot 081819190842622$ for WGS 84 (NIMA, 2000) and $a_{e}=3437 \cdot 74677078$ nautical miles (nm) (Bowditch, 2002).

According to the formulae of the Mercator sailing,

$$
\begin{gathered}
\ell=L_{\mathrm{X}_{i+1}}-L_{\mathrm{X}_{i}}, d l o=\lambda_{\mathrm{X}_{i+1}}-\lambda_{\mathrm{X}_{i}}, m=M_{\mathrm{X}_{i+1}}-M_{\mathrm{X}_{i}}, \\
\tan c m=\frac{d l o \times 60^{\prime}}{m}, \\
d m= \begin{cases}\ell \sec c m, & c m \neq 90^{\circ} \\
d l o \cos L_{\mathrm{X}_{i}}, & c m=90^{\circ}\end{cases}
\end{gathered}
$$

\subsection{Computation procedures for the simplified GCEM and the COFI method.}

- Step 1. Great circle information.

1. Calculate the great circle distance and initial course angle by using Equations (15) and (17), respectively.

2. Calculate the equator crossing point by using Equation (39).

3. Calculate the vertex by using Equations (40) and (41).

- Step 2. Waypoints along the GCT.

Condition 1. Use the longitudes of the waypoints to obtain the latitudes by using Equation (38).

Condition 2. Use the great circle distances from the departure to yield the latitudes and longitudes of the waypoints by using Equations (19) and (21).

- Step 3. According to Mercator sailing, the Mercator course and distance of every rhumb line between two adjacent waypoints can be determined by using Equations (42), (43), (44) and (45).

3.2. Developing the numerical program. A GCS program, called "GCSPro", covering Conditions 1 and 2, has been developed based on the COFI method and the simplified GCEM. For ease of use, GCSPro uses Visual Basic (VB) with a graphical 
Table 2. Results of solving waypoints along the GCT under $\lambda_{X}$ by using the STM-E in Example 1.

\begin{tabular}{|c|c|c|c|c|}
\hline \multirow[b]{2}{*}{ Process } & \multicolumn{4}{|l|}{ Item } \\
\hline & Equation & Input & Output & Solution \\
\hline $\begin{array}{l}1 \\
2\end{array}$ & $\begin{array}{l}(1) \\
(2)\end{array}$ & $\begin{array}{l}L_{\mathrm{F}}=40^{\circ} 27 \cdot 0^{\prime}(\mathrm{N}) \\
L_{\mathrm{T}}=-34^{\circ} 25 \cdot 0^{\prime}(\mathrm{S}) \\
D L o=092^{\circ} \mathrm{E}\end{array}$ & $\begin{aligned} D & =112 \cdot 867789^{\circ} \\
C & =-63 \cdot 479168^{\circ} \\
& =116 \cdot 520832^{*}\end{aligned}$ & $\begin{array}{l}D \fallingdotseq 6772 \cdot 1^{\prime} \\
\mathrm{N} 116 \cdot 5^{\circ} \mathrm{E} \\
C_{n}=116 \cdot 5^{\circ}\end{array}$ \\
\hline 4 & $\begin{array}{l}\text { (3) } \\
\text { (4) }\end{array}$ & $\begin{array}{l}L_{\mathrm{F}}=40^{\circ} 27 \cdot 0^{\prime} \\
C=116 \cdot 520832^{\circ} \\
\lambda_{\mathrm{F}}=073^{\circ} 50 \cdot 0^{\prime} \mathrm{W}\end{array}$ & $\begin{array}{l}D L o_{\mathrm{FE}}=52 \cdot 43302404^{\circ} \mathrm{E} \\
C_{\mathrm{E}}=42 \cdot 91376773^{\circ}\end{array}$ & $\lambda_{\mathrm{E}}=021^{\circ} 24 \cdot 0^{\prime} \mathrm{W}$ \\
\hline \multirow{8}{*}{5} & \multirow{8}{*}{ (5) } & $\begin{aligned} D L o_{\mathrm{E} X} & =021^{\circ} 24 \cdot 0^{\prime} \mathrm{W} \sim 060^{\circ} \mathrm{W} \\
& =38^{\circ} 36 \cdot 0^{\prime} \mathrm{W}\end{aligned}$ & $L_{X}=33^{\circ} 51 \cdot 8^{\prime}$ & $\left(33^{\circ} 51 \cdot 8^{\prime} \mathrm{N}, 060^{\circ} \mathrm{W}\right)$ \\
\hline & & $D L o_{\mathrm{E} X}=28^{\circ} 36 \cdot 0^{\prime} \mathrm{W}$ & $L_{X}=27^{\circ} 14 \cdot 6^{\prime}$ & $\left(27^{\circ} 14 \cdot 6^{\prime} \mathrm{N}, 050^{\circ} \mathrm{W}\right)$ \\
\hline & & $D L o_{\mathrm{E} X}=18^{\circ} 36 \cdot 0^{\prime} \mathrm{W}$ & $L_{X}=18^{\circ} 56 \cdot 2^{\prime}$ & $\left(18^{\circ} 56 \cdot 2^{\prime} \mathrm{N}, 040^{\circ} \mathrm{W}\right)$ \\
\hline & & $D L o_{\mathrm{E} X}=08^{\circ} 36 \cdot 0^{\prime} \mathrm{W}$ & $L_{X}=09^{\circ} 08 \cdot 2^{\prime}$ & $\left(09^{\circ} 08 \cdot 2^{\prime} \mathrm{N}, 030^{\circ} \mathrm{W}\right)$ \\
\hline & & $D L o_{\mathrm{E} X}=01^{\circ} 24 \cdot 0^{\prime} \mathrm{W}$ & $L_{X}=-01^{\circ} 30 \cdot 3^{\prime}$ & $\left(01^{\circ} 30 \cdot 3^{\prime} \mathrm{S}, 020^{\circ} \mathrm{W}\right)$ \\
\hline & & $D L o_{\mathrm{E} X}=11^{\circ} 24 \cdot 0^{\prime} \mathrm{E}$ & $L_{X}=-12^{\circ} 00 \cdot 2^{\prime}$ & $\left(12^{\circ} 00 \cdot 2^{\prime} \mathrm{S}, 010^{\circ} \mathrm{W}\right)$ \\
\hline & & $D L o_{\mathrm{E} X}=21^{\circ} 24 \cdot 0^{\prime} \mathrm{E}$ & $L_{X}=-21^{\circ} 25 \cdot 7^{\prime}$ & $\left(21^{\circ} 25 \cdot 7^{\prime} \mathrm{S}, 000^{\circ}\right)$ \\
\hline & & $D L o_{\mathrm{E} X}=31^{\circ} 24 \cdot 0^{\prime} \mathrm{E}$ & $L_{X}=-29^{\circ} 16 \cdot 0^{\prime}$ & $\left(29^{\circ} 16^{\circ} 0^{\prime} \mathrm{S}, 010^{\circ} \mathrm{E}\right)$ \\
\hline
\end{tabular}

* Since $\tan (-\theta)=\tan \left(180^{\circ}-\theta\right),(-\theta)$ is replaced as $\left(180^{\circ}-\theta\right)$.

user interface (GUI) for programming. In addition, for the purpose of choosing reasonable numbers of the waypoints, a diagram of total Mercator distance versus waypoints number (called tMd-n diagram) is provided for the navigator.

\section{DEMONSTRATED EXAMPLES AND DISCUSSION.}

4.1. Example 1. A vessel is proceeding from New York (USA) to Cape Town (South Africa). The master desires to use the great circle sailing from $L 40^{\circ} 27 \cdot 0^{\prime} \mathrm{N}, \lambda 073^{\circ}$ $50 \cdot 0^{\prime} \mathrm{W}$ to $L 34^{\circ} 25 \cdot 0^{\prime} \mathrm{S}, \lambda 018^{\circ} 10 \cdot 0^{\prime} \mathrm{E}$.

4.1.1. Required. Calculate the following cases under different given conditions by using the STM-E.

1. Calculate the great circle distance, initial course and the latitudes and longitudes of the waypoints along the GCT at longitude $060^{\circ} \mathrm{W}$ and at each $10^{\circ}$ of longitude thereafter to longitude $010^{\circ} \mathrm{E}$ (Condition 1).

2. Calculate the latitudes and longitudes of the waypoints along the GCT at equal interval of great circle distance, $600\left(10^{\circ}\right)$ nautical miles $(\mathrm{nm})$, from the equator crossing point (Condition 2).

\subsubsection{Solution.}

1. The STM-E is adopted to solve the waypoints along the GCT under Condition 1. Results and the solving procedures with the suggested formulae are shown in Table 2. 
Table 3. Results of solving waypoints along the GCT under $D_{\mathrm{E} X}$ by using the STM-E in Example 1.

\begin{tabular}{|c|c|c|c|c|}
\hline \multirow[b]{2}{*}{ Process } & \multicolumn{4}{|l|}{ Item } \\
\hline & Equation & Input & Output & Solution \\
\hline & \multicolumn{4}{|c|}{$\begin{array}{l}\text { Process } 1-4 \text { is the same as Table } 2 . \\
\text { Preliminary: } \lambda_{\mathrm{E}}=021^{\circ} 24 \cdot 0^{\prime} \mathrm{W} C_{\mathrm{E}}=42 \cdot 91376773^{\circ}\end{array}$} \\
\hline $\begin{array}{l}5 \\
6\end{array}$ & $\begin{array}{l}(6) \\
(7)\end{array}$ & $D_{\mathrm{E} X}=00^{\circ}$ & $\begin{array}{l}L_{X}=00^{\circ}=L_{\mathrm{E}} \\
D L o_{\mathrm{E} X}=00^{\circ}\left(\lambda_{X}=\lambda_{\mathrm{E}}\right)\end{array}$ & $\left(00^{\circ}, 021^{\circ} 24 \cdot 0^{\prime} \mathrm{W}\right)$ \\
\hline $\begin{array}{l}5 \\
6\end{array}$ & $\begin{array}{l}(6) \\
(7)\end{array}$ & $D_{\mathrm{E} X}=10^{\circ}$ & $\begin{array}{l}L_{X}= \pm 07^{\circ} 18 \cdot 4^{\prime} \\
D L o_{\mathrm{E} X}=06^{\circ} 50 \cdot 8^{\prime}(\mathrm{W} / \mathrm{E})\end{array}$ & $\begin{array}{l}\left(07^{\circ} 18 \cdot 4^{\prime} \mathrm{N}, 028^{\circ} 14 \cdot 8^{\prime} \mathrm{W}\right) \\
\left(07^{\circ} 18 \cdot 4^{\prime} \mathrm{S}, 014^{\circ} 33 \cdot 2^{\prime} \mathrm{W}\right)\end{array}$ \\
\hline $\begin{array}{l}5 \\
6\end{array}$ & $\begin{array}{l}(6) \\
(7)\end{array}$ & $D_{\mathrm{E} X}=20^{\circ}$ & $\begin{array}{l}L_{X}= \pm 14^{\circ} 30 \cdot 4^{\prime} \\
D L o_{\mathrm{E} X}=13^{\circ} 55 \cdot 1^{\prime}(\mathrm{W} / \mathrm{E})\end{array}$ & $\begin{array}{l}\left(14^{\circ} 30 \cdot 4^{\prime} \mathrm{N}, 035^{\circ} 19 \cdot 1^{\prime} \mathrm{W}\right) \\
\left(14^{\circ} 30 \cdot 4^{\prime} \mathrm{S}, 007^{\circ} 28 \cdot 9^{\prime} \mathrm{W}\right)\end{array}$ \\
\hline $\begin{array}{l}5 \\
6\end{array}$ & $\begin{array}{l}(6) \\
(7)\end{array}$ & $D_{\mathrm{E} X}=30^{\circ}$ & $\begin{array}{l}L_{X}= \pm 21^{\circ} 28 \cdot 8^{\prime} \\
D L o_{\mathrm{E} X}=21^{\circ} 27 \cdot 6^{\prime}(\mathrm{W} / \mathrm{E})\end{array}$ & $\begin{array}{l}\left(21^{\circ} 28 \cdot 8^{\prime} \mathrm{N}, 042^{\circ} 51 \cdot 6^{\prime} \mathrm{W}\right) \\
\left(21^{\circ} 28 \cdot 8^{\prime} \mathrm{S}, 000^{\circ} 03 \cdot 6^{\prime} \mathrm{E}\right)\end{array}$ \\
\hline $\begin{array}{l}5 \\
6\end{array}$ & $\begin{array}{l}(6) \\
(7)\end{array}$ & $D_{\mathrm{E} X}=40^{\circ}$ & $\begin{array}{l}L_{X}= \pm 28^{\circ} 05 \cdot 0^{\prime} \\
D L o_{\mathrm{E} X}=29^{\circ} 44 \cdot 5^{\prime}(\mathrm{W} / \mathrm{E})\end{array}$ & $\begin{array}{l}\left(28^{\circ} 0 \cdot 0^{\prime} \mathrm{N}, 051^{\circ} 08 \cdot 5^{\prime} \mathrm{W}\right) \\
\left(28^{\circ} 05 \cdot 0^{\prime} \mathrm{S}, 008^{\circ} 20 \cdot 5^{\prime} \mathrm{E}\right)\end{array}$ \\
\hline $\begin{array}{l}5 \\
6\end{array}$ & $\begin{array}{l}(6) \\
(7)\end{array}$ & $D_{\mathrm{E} X}=50^{\circ}$ & $\begin{array}{l}L_{X}= \pm 34^{\circ} 07 \cdot 6^{\prime} \\
D L o_{\mathrm{E} X}=39^{\circ} 03 \cdot 5^{\prime}(\mathrm{W} / \mathrm{E})\end{array}$ & $\begin{array}{l}\left(34^{\circ} 07 \cdot 6^{\prime} \mathrm{N}, 060^{\circ} 27 \cdot 5^{\prime} \mathrm{W}\right) \\
\left(34^{\circ} 07 \cdot 6^{\prime} \mathrm{S}, 017^{\circ} 39 \cdot 5^{\prime} \mathrm{E}\right)\end{array}$ \\
\hline $\begin{array}{l}5 \\
6\end{array}$ & $\begin{array}{l}\text { (6) } \\
(7)\end{array}$ & $D_{\mathrm{E} X}=60^{\circ}$ & $\begin{array}{l}L_{X}= \pm 39^{\circ} 21 \cdot 9^{\prime} \\
D L o_{\mathrm{E} X}=49^{\circ} 42 \cdot 3^{\prime}(\mathrm{W})\end{array}$ & $\left(39^{\circ} 31 \cdot 9^{\prime} \mathrm{N}, 071^{\circ} 06 \cdot 3^{\prime} \mathrm{W}\right)$ \\
\hline
\end{tabular}

\begin{tabular}{|c|c|c|c|c|c|}
\hline GCSPro & & & & & 0日 \\
\hline Great Circle Information & Way & & & & \\
\hline The great circle initial course is $240.3^{\circ}\left(\mathrm{N} 119.7^{\circ} \mathrm{W}\right.$ & WP. & Latitude & Longitude & Course & Distance \\
\hline The great circle distance is 6445.22 & $\mathrm{~F}$ & $37^{\circ} 47.5^{\prime} \mathrm{N}$ & $122^{\circ} 27.8^{\prime} \mathrm{W}$ & $238.5^{\circ}$ & 361.21 \\
\hline The total Mercator distance is $6466.03^{\prime}$ & 1 & $34^{\circ} 38.7^{7} \mathrm{~N}$ & $128^{\circ} 47.9^{\prime} \mathrm{W}$ & $235.0^{\circ}$ & $361.19^{\prime}$ \\
\hline The difference between great circle and Mercator distance is $20.80^{\circ}$ & 2 & $31^{\circ} 11.7 \mathrm{~N}$ & $134^{\circ} 39.0^{\prime} \mathrm{W}$ & $232.1^{\circ}$ & $361.18^{\prime}$ \\
\hline & 3 & $27^{\circ} 30.0^{\circ} \mathrm{N}$ & $140^{\circ} 4.5^{\prime} \mathrm{W}$ & $229.7^{\circ}$ & 361.17 \\
\hline The equator crossing points: & 4 & $23^{\circ} 36.6^{\prime} \mathrm{N}$ & $145^{\circ} 8.4^{\prime} \mathrm{W}$ & $227.8^{\circ}$ & 361.16 \\
\hline$\left(0,169^{\circ} 30^{\top} \mathrm{W}\right)\left(0,10^{\circ} 30 \mathrm{E}\right)$ & 5 & $19^{\circ} 33.8^{\circ} \mathrm{N}$ & $149^{\circ} 54.4^{\prime} \mathrm{W}$ & 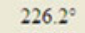 & $361.15^{\prime}$ \\
\hline One of the equator crossing points on the $\mathrm{GC}$ track. & 6 & $15^{\circ} 23.9^{\prime} \mathrm{N}$ & $154^{\circ} 26.1^{\prime} \mathrm{W}$ & $225.0^{\circ}$ & $361.15^{\prime}$ \\
\hline & 7 & $11^{\circ} 8.7^{\prime} \mathrm{N}$ & $158^{\circ} 47.1^{\prime} \mathrm{W}$ & 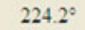 & $361.15^{\prime}$ \\
\hline The vertexes: & 8 & $6^{\circ} 49.9^{\prime} \mathrm{N}$ & $163^{\circ} .5^{\prime} \mathrm{W}$ & $223.7^{\circ}$ & $361.15^{\prime}$ \\
\hline$\left(46^{\circ} 39.5^{\prime} \mathrm{N}, 79^{\circ} 30^{\prime} \mathrm{W}\right)\left(46^{\circ} 39.5^{\prime} \mathrm{S}, 100^{\circ} 30^{\prime} \mathrm{E}\right)$ & 9 & $2^{\circ} 28.9^{\prime} \mathrm{N}$ & $167^{\circ} 9.3^{\prime} \mathrm{W}$ & $223.5^{\circ}$ & $361.14^{\prime}$ \\
\hline The vertexes out of the GC track. & 10 & $1^{\circ} 52.9^{\prime} \mathrm{S}$ & $171^{\circ} 16.6 \mathrm{~W}$ & $223.7^{\circ}$ & $361.15^{\prime}$ \\
\hline & 11 & $6^{\circ} 14.0^{\prime} \mathrm{S}$ & $175^{\circ} 25.0^{\prime} \mathrm{W}$ & $224.1^{\circ}$ & $361.15^{\prime}$ \\
\hline & 12 & $10^{\circ} 33.2^{\prime} \mathrm{S}$ & $179^{\circ} 37.6 \cdot \mathrm{W}$ & $224.9^{\circ}$ & 361.15 \\
\hline Total Mercator distance $(\mathrm{nm}$. $)$ & 13 & $14^{\circ} 49.0^{\prime} \mathrm{S}$ & $176^{\circ} 2.7^{\prime} \mathrm{E}$ & $226.0^{\circ}$ & $\begin{array}{l}361.15^{\prime} \\
361.16\end{array}$ \\
\hline & $\begin{array}{l}14 \\
15\end{array}$ & $\begin{array}{r}18^{\circ} 59.7^{\prime} \mathrm{S} \\
23^{\circ} 3.6^{\prime} \mathrm{S}\end{array}$ & $\begin{array}{l}171^{\circ} 32.6^{\prime} \mathrm{E} \\
166^{\circ} 4888^{\prime} \mathrm{E}\end{array}$ & $\begin{array}{l}227.5^{\circ} \\
229.4^{\circ}\end{array}$ & $\begin{array}{l}361.16^{\prime} \\
361.17\end{array}$ \\
\hline 6484.600 & 16 & $26^{\circ} 58.5^{\prime} \mathrm{S}$ & $161^{\circ} 47.6^{\circ} \mathrm{E}$ & $231.8^{\circ}$ & $361.18^{\prime}$ \\
\hline & 17 & $30^{\circ} 42.0^{\prime} \mathrm{S}$ & $156^{\circ} 25.3^{\prime} \mathrm{E}$ & $234.5^{\circ}$ & $326.29^{\prime}$ \\
\hline & $\mathrm{T}$ & $33^{\circ} 51.7^{\prime} \mathrm{S}$ & $151^{\circ} 12.7^{\prime} \mathrm{E}$ & & \\
\hline 6445.22 & & & & & \\
\hline $5 \quad 10 \quad 15$ & & & & & \\
\hline
\end{tabular}

Figure 5. Results of running the GCSPro under Condition 2 in Example 2. 
Table 4. A comparison of results obtained by the Ageton method and the COFI method in Example 2.

\begin{tabular}{lll}
\hline Item & \multicolumn{1}{c}{ The Ageton method* } & \multicolumn{1}{c}{ The COFI method } \\
\hline$D$ & $644 \cdot 5^{\prime}$ & $6445 \cdot 22^{\prime}$ \\
$C$ & $240^{\circ} 17 \cdot 5^{\prime}$ & $240 \cdot 3^{\circ}$ \\
$V$ & $L 46^{\circ} 39 \cdot 5^{\prime} \mathrm{S}, \lambda 100^{\circ} 29 \cdot 7^{\prime} \mathrm{E}$ & $L 46^{\circ} 39 \cdot 5^{\prime} \mathrm{S}, \lambda 100^{\circ} 30 \cdot 0^{\prime} \mathrm{E}$ \\
$D_{\mathrm{F} X}=6^{\circ}$ & $L 34^{\circ} 39 \cdot 0^{\prime} \mathrm{N}, \lambda 128^{\circ} 48 \cdot 3^{\prime} \mathrm{W}$ & $L 34^{\circ} 38 \cdot 7^{\prime} \mathrm{N}, \lambda 128^{\circ} 47 \cdot 9^{\prime} \mathrm{W}$ \\
$D_{\mathrm{F} X}=12^{\circ}$ & $L 31^{\circ} 12 \cdot 0^{\prime} \mathrm{N}, \lambda 134^{\circ} 39 \cdot 3^{\prime} \mathrm{W}$ & $L 31^{\circ} 11 \cdot 7^{\prime} \mathrm{N}, \lambda 134^{\circ} 39 \cdot 0^{\prime} \mathrm{W}$ \\
$D_{\mathrm{F} X}=18^{\circ}$ & $L 27^{\circ} 30 \cdot 0^{\prime} \mathrm{N}, \lambda 140^{\circ} 04 \cdot 3^{\prime} \mathrm{W}$ & $L 27^{\circ} 30 \cdot 0^{\prime} \mathrm{N}, \lambda 140^{\circ} 04 \cdot 5^{\prime} \mathrm{W}$ \\
$D_{\mathrm{F} X}=36^{\circ}$ & $L 15^{\circ} 24 \cdot 0^{\prime} \mathrm{N}, \lambda 154^{\circ} 26 \cdot 3^{\prime} \mathrm{W}$ & $L 15^{\circ} 23 \cdot 9^{\prime} \mathrm{N}, \lambda 154^{\circ} 26 \cdot 1^{\prime} \mathrm{W}$ \\
$D_{\mathrm{F} X}=54^{\circ}$ & $L 02^{\circ} 29 \cdot 0^{\prime} \mathrm{N}, \lambda 167^{\circ} 09 \cdot 3^{\prime} \mathrm{W}$ & $L 02^{\circ} 28^{\prime} \cdot 9^{\prime} \mathrm{N}, \lambda 167^{\circ} 09 \cdot 3^{\prime} \mathrm{W}$ \\
$D_{\mathrm{F} X}=60^{\circ}$ & $L 01^{\circ} 52 \cdot 5^{\prime} \mathrm{N}, \lambda 171^{\circ} 17 \cdot 3^{\prime} \mathrm{W}$ & $L 01^{\circ} 52^{\prime} \cdot 9^{\prime} \mathrm{S}, \lambda 171^{\circ} 16 \cdot 6^{\prime} \mathrm{W}$ \\
\hline
\end{tabular}

* Resource: Bowditch, 1981, P.616-618.

Table 5. The relationship between total Mercator distance $(\mathrm{nm})$ and waypoint number on the GCT in Example 2.

\begin{tabular}{lccc}
\hline Waypoints no. & Total Mercator distance & Waypoints no. & Total Mercator distance \\
\hline 0 & $6484 \cdot 60$ & 9 & $6466 \cdot 74$ \\
1 & $6483 \cdot 89$ & 10 & $6466 \cdot 56$ \\
2 & $6475 \cdot 60$ & 11 & $6466 \cdot 43$ \\
3 & $6471 \cdot 67$ & 12 & $6466 \cdot 32$ \\
4 & $6469 \cdot 65$ & 13 & $6466 \cdot 24$ \\
5 & $6468 \cdot 49$ & 14 & $6466 \cdot 17$ \\
6 & $6467 \cdot 77$ & 15 & $6466 \cdot 12$ \\
7 & $6467 \cdot 30$ & 16 & $6466 \cdot 07$ \\
8 & $6466 \cdot 97$ & 17 & $6466 \cdot 03$ \\
\hline
\end{tabular}

2. The STM-E is adopted to solve the waypoints along the GCT under Condition 2. Results and the solving procedures with the suggested formulae are shown in Table 3 .

4.1.3. Discussion. In this example, although the STM-E can solve waypoints problems under Conditions 1 and 2, many sign convention judgments arise in the solving procedure of the STM-E. Anyway, it still offers another solving approach for the IA.

4.2. Example 2. A vessel is proceeding from San Francisco (USA) to Sydney (Australia). The navigator desires to use great circle sailing from $L 37^{\circ} 47 \cdot 5^{\prime} \mathrm{N}, \lambda 122^{\circ}$ $27 \cdot 8^{\prime} \mathrm{W}$ to ${\mathrm{L} 33^{\circ}}^{\circ} 51 \cdot 7^{\prime} \mathrm{S}, \lambda 151^{\circ} 12 \cdot 7^{\prime} \mathrm{E}$ (Bowditch, 1981, P.616-618).

4.2.1. Required. Using GCSPro to calculate the latitudes and longitudes of the waypoints on the GCT $360 \mathrm{~nm}$ apart (Condition 2), and the information for the great circle (eg. the great circle distance, initial course, the equator crossing point and the vertices).

4.2.2. Solution. GCSPro is used to solve the waypoints on the GCT under Condition 2. Results including of the waypoints, great circle information, and a tMd-n diagram are shown in Figure 5. The comparison of results obtained by the COFI method and those by the Ageton method (tabular method) is shown in Table 4. In addition, when the "show data" button in Figure 5 is clicked, detailed numerical 


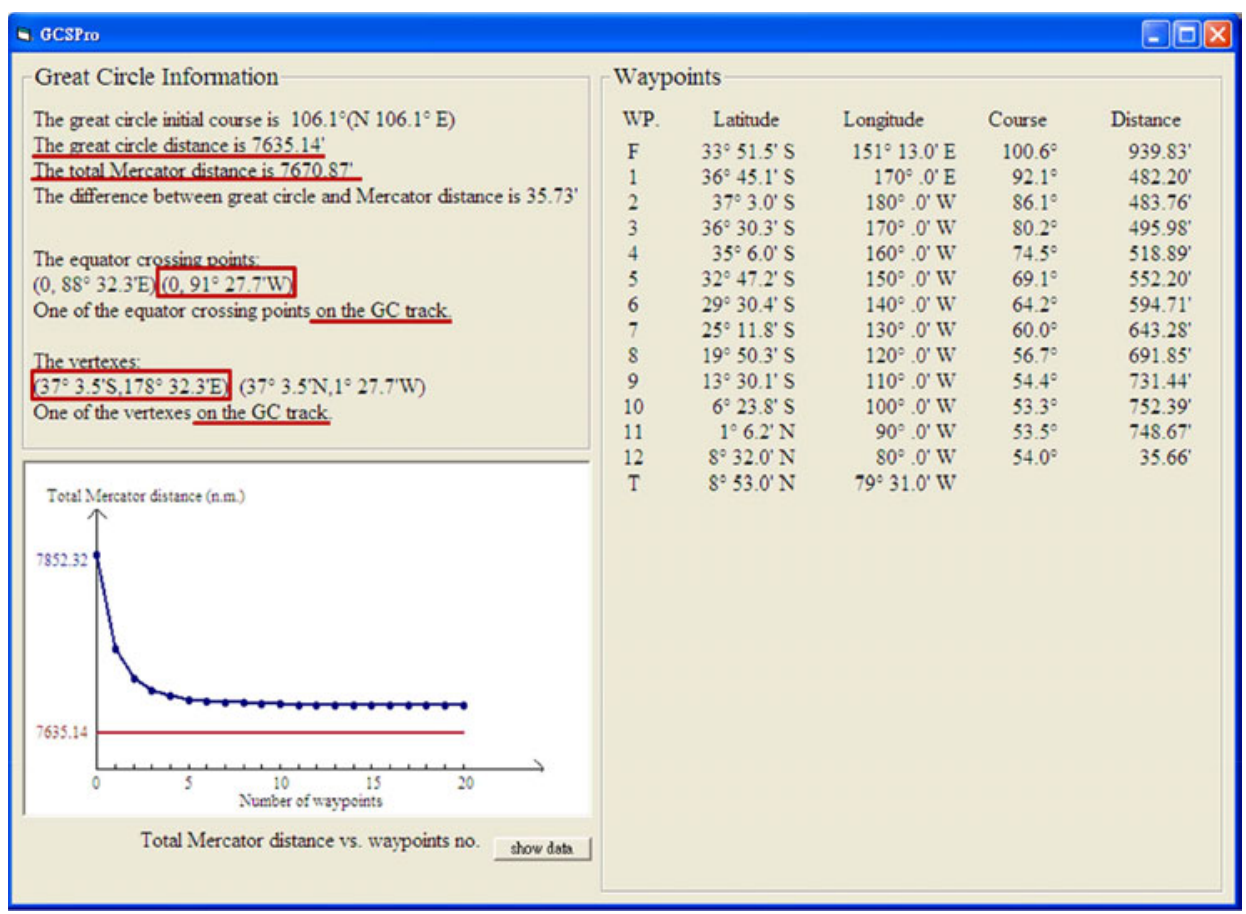

Figure 6. Results of running the GCSPro under Condition 1 in Example 3.

information of total Mercator distance versus numbers of waypoints will display in the format shown in Table 5 .

4.2.3. Discussion. In this example, the COFI method has been validated successfully. In Table 4, it is found that the COFI method is more accurate than the Ageton method because the former is free of rounding errors, which was also reported in Bowditch (2002). As for Table 5, the total Mercator distance of 16 waypoints is nearly equal to that of 8 waypoints and their distance difference is less than $1 \mathrm{~nm}$. An optimum number of waypoints can be determined from this table for the navigator.

4.3. Example 3. A vessel is proceeding from Sydney (Australia) to Balboa (Panama). The master desires to use the great circle sailing from L33 ${ }^{\circ} 51 \cdot 5^{\prime} \mathrm{S}, \lambda 151^{\circ}$ $13 \cdot 0^{\prime} \mathrm{E}$, to $L 08^{\circ} 53 \cdot 0^{\prime} \mathrm{N}, \lambda 079^{\circ} 31 \cdot 0^{\prime} \mathrm{W}$ (Chen et al, 2004. pp. 317-319).

4.3.1. Required. Using GCSPro to calculate the latitudes and longitudes of the waypoints on the GCT at longitude and at each 10 degrees of longitude thereafter to longitude (Condition 1), and the information for the great circle (eg. the great circle distance, initial course, the equator crossing point and the vertices).

4.3.2. Solution. GCSPro is used to solve the waypoints along the GCT under Condition 1 successfully. Results including the waypoints, great circle information and a tMd-n diagram are shown in Figure 6.

4.3.3. Discussion. Based on the proposed COFI method and the simplified GCEM, the developed GCSPro program has been validated by Examples 2 and 3. It is found that the GCSPro program shows the advantages of completeness and practical application. 
5. CONCLUSIONS. In this paper, the COFI method has been developed to calculate the waypoints along the GCT successfully using the multiple products of the vector algebra (VA-MP). Due to fixing the initial great circle course, the GCT can be determined and the waypoints along the GCT can be calculated directly. In addition, without tedious derivations, the COFI method is simpler and more straightforward than the conventional methods. A program, GCSPro, for calculating GCT problems has been validated by the practical examples. It is found that the program can be user friendly and effectively operated by the navigator under two given initial conditions. Finally, the STM-E has been derived and also offers another way to solve the waypoints problem successfully.

\section{ACKNOWLEDGEMENT}

Constructive suggestions by Dr. Jiang-Ren Chang are highly appreciated. In addition, financial support from the National Science Council, Taiwan under contract number: NSC 95-2221-E019-092- and NSC 96-2628-E-019-022-MY3 are acknowledged.

\section{REFERENCES}

Bowditch, N. (1981). American Practical Navigator, DMAH/TC, Washington.

Bowditch, N. (2002). The American Practical Navigator, 2002 Bicentennial Edition. National Imagery and Mapping Agency (NIMA).

Chen, C. L., Hsu, T. P. and Chang, J. R. (2004). A Novel Approach to Great Circle Sailings: The Great Circle Equation. The Journal of Navigation, 57(2), 311-320.

Clough-Smith, J. H. (1966). An Introduction to Spherical Trigonometry, Brown, Son \& Ferguson, Ltd., Glasgow.

Cutler, T.J. (2004). Dutton's Nautical Navigation, Fifteenth Edition. Naval Institute Press, Maryland.

Holm, R. J. (1972). Great Circle Waypoints for Inertial Equipped Aircraft. Journal of the Institute of Navigation, 19(2), 191-194.

Jofeh, M. L. (1981). The Analysis of Great-circle Tracks. The Journal of Navigation, 34(1), 148-149.

Keys, G. (1983). Practical Navigation by Calculator, Stanford Maritime, London.

Miller, A. R., Moskowitz, I. S. and Simmen, J. (1991). Traveling on the Curved Earth. Journal of the Institute of Navigation, 38(1), 71-78.

Nastro, V. and Tancredi, U. (2010). Great Circle Navigation with Vectorial Methods. The Journal of Navigation, 63(3), 557-563.

National Imagery and Mapping Agency (NIMA) (2000). Department of Defense World Geodetic System 1984: Its definition and relationship with local geodetic systems, Technical Report NIMA TR8350·2., Third Edition. NIMA.

Spiegel, M. R., Lipschutz, S. and Spellman, D. (2009). Vector Analysis and an Introduction to Tensor Analysis, Second Edition. McGraw-Hill, NY, USA.

\section{APPENDIX}

$\begin{array}{ll}\mathrm{F} & \text { the departure } \\ \mathrm{T} & \text { the destination } \\ \mathrm{P}_{\mathrm{nr}} & \text { the pole nearer the departure in Figures 1,2 and } 3 \\ \mathrm{P} & \text { the North Pole } \\ \mathrm{E} & \text { the equator crossing point of the great circle } \\ \mathrm{V} & \text { the vertex of the great circle } \\ X ; X i & \text { The waypoints along the GCT }\end{array}$




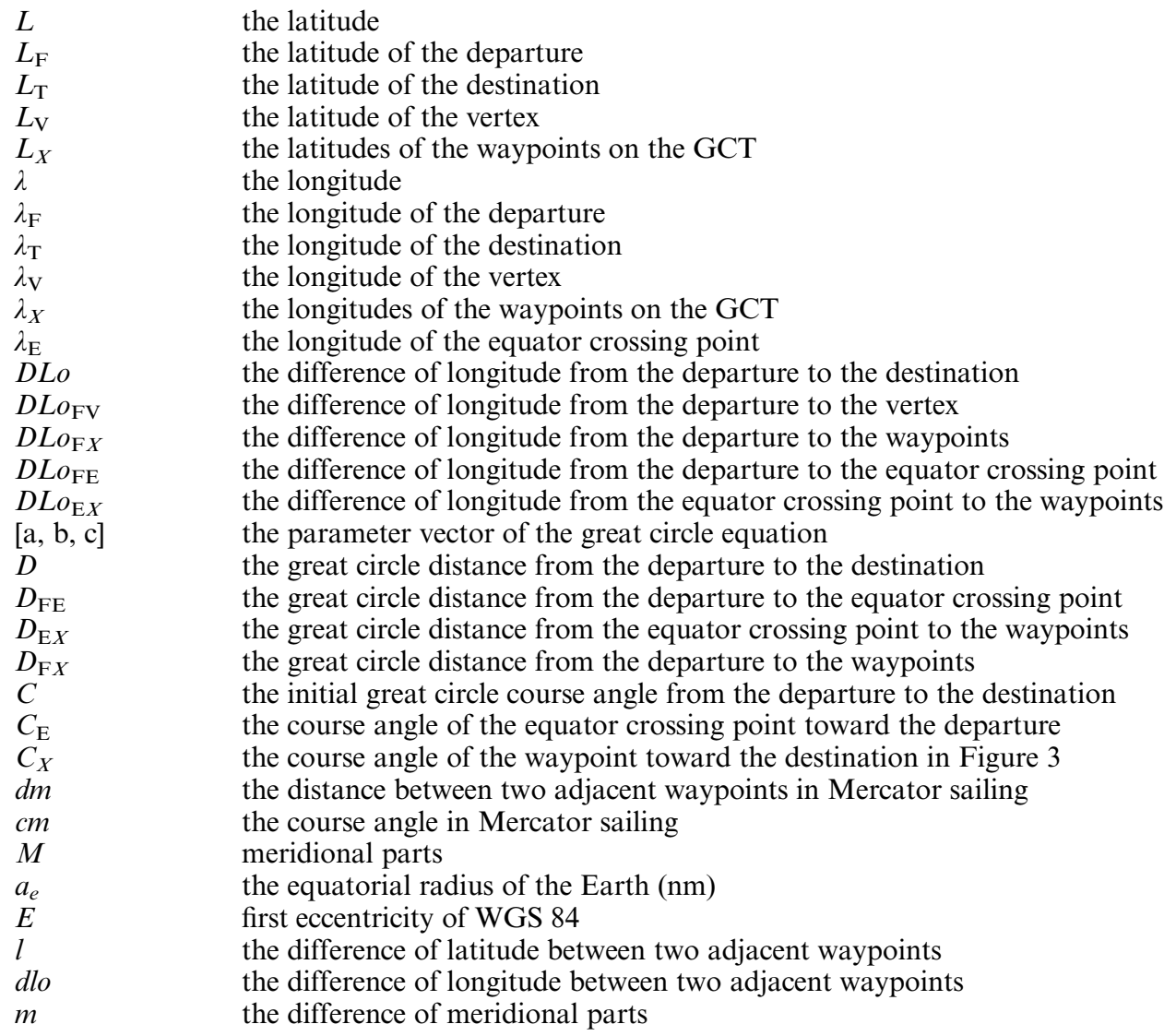

